\title{
Creatividad y enfoques de aprendizaje en estudiantes universitarios
}

\section{Creativity and learning approaches in college students}

\author{
Agustín Freiberg-Hoffmann (iD - Carlos Vigh (iD - Mercedes Fernández-Liporace \\ Universidad de Buenos Aires - Consejo Nacional de Investigaciones Científicas y Técnicas \\ (CONICET). Buenos Aires, Argentina.
}

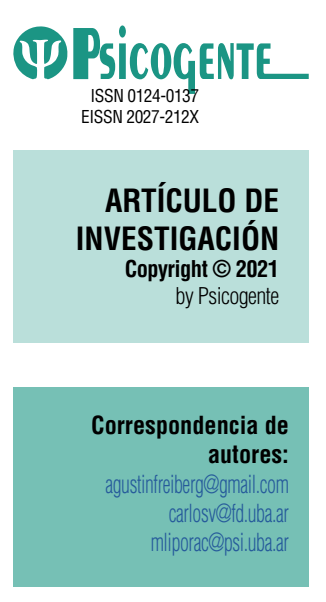

Recibido: 09-09-20 Aceptado: $07-07-21$ Publicado: $30-07-21$

\section{Resumen}

Introducción: Mejorar el aprendizaje de los estudiantes y adaptarlo a las demandas del mercado laboral actual es un tema de gran interés planteado por distintos organismos internacionales. Se busca así que los alumnos alcancen un aprendizaje profundo de los contenidos académicos y que a su vez desarrollen su capacidad creativa para la resolución de problemas.

Objetivo: Analizar los enfoques de aprendizaje -Profundo y Superficial- y la creatividad -proceso creativo y personalidad creativa- en estudiantes universitarios según variables sociodemográficas y académicas.

Método: Se empleó un diseño no experimental y transversal. Se respondió a un objetivo de diferencias entre grupos y a otro explicativo. Participaron 301 estudiantes universitarios de Psicología (51 \%) e Ingeniería (49\%).

Resultados: Se registraron diferencias significativas en los enfoques profundo y superficial: el primero a favor de las mujeres, y el segundo favorable a los varones. En relación con la creatividad, se observó una diferencia significativa en la personalidad creativa a favor de los varones, y del proceso creativo a favor de los estudiantes de Psicología. Por otra parte, el rendimiento académico fue explicado negativamente por el enfoque Superficial $(\beta=-0,276)$ y positivamente, por el proceso creativo $(\beta=0,185)$.

Conclusiones: Para mejorar el rendimiento académico se necesita aplicar estrategias de enseñanza y evaluación que desestimulen el aprendizaje Superficial y promuevan el proceso creativo en los estudiantes. Los cambios en las prácticas educativas deberán, además, enfocarse en mejorar el aprendizaje Profundo y la creatividad para ajustar el perfil profesional de habilidades de los egresados al requerido actualmente por el ámbito laboral.

Palabras clave: proceso creativo, personalidad creativa, estudiantes universitarios, rendimiento académico, éxito académico.

\section{Abstract}

Introduction: International organizations have drawn attention to the idea of adapting learning to fit the graduates' skills and competences to the current requirements of labor market. Therefore, reaching a deep learning of academic contents along with developing creative ability for problem solving emerges as a desirable goal to be reached.

Objective: The study was aimed at analyzing the Deep and Surface learning approaches as well as creativity -comprising the creative process and the creative personality- by socio-demographic and academic variables in college students.

Method: A non-experimental and cross-sectional design was conducted, entailing two studies: a group differences one and another, explanatory. The sample was composed of 301 college students attending two majors -Psychology (51 \%) and Engineering (49\%).

Results: Significant differences in the use of learning approaches were found; women used the Deep approach more often whereas men chose the Surface approach. As for creativity, men showed statistically significant differences in creative personality over women. The creative process arose more often in Psychology students when compared with Engineering undergraduates, with significant $p$-values. Academic Perfomance was negatively explained by the Surface approach $(\beta=-0,276)$ and positively, by the creative process $(\beta=0,185)$.

Conclusions: Boosting Academic Perfomance requires a major adjustment in teaching and evaluation methods. They should be aimed at discouraging the use of the Surface approach whilst promoting the 

current labor market requirements.

Keywords: creative process, creative personality, college students, academic performance, academic success.

\section{INTRODUCCIÓN}

El sistema universitario argentino actual enfrenta el desafío de mejorar la calidad de los aprendizajes y el rendimiento académico de los estudiantes. Existen dos grandes indicadores que permiten conocer la situación del sistema universitario que son la equidad y la calidad. La equidad remite a la igualdad en cuanto a acceso y recursos de todos los estudiantes. Este indicador se puede medir mediante el porcentaje del producto bruto interno que destinan los gobiernos a la educación, otorgamiento de notebooks y becas a los alumnos y la tasa de ingresantes al sistema, entre otras cosas (UNESCO, 2013). La calidad, por su parte, puede definirse de cinco modos distintos que son: como exclusividad, perfección, objetivos alcanzados, producto económico, y transformación (Harvey \& Green, 1993). La tercera noción -objetivos alcanzadosse relaciona con el concepto de rendimiento académico, y es la adoptada en general por los gobiernos a la hora de ponderar la calidad educativa y asignar, con base en estos datos, las partidas presupuestarias. En Argentina, se exige a los estudiantes como rendimiento mínimo la aprobación de dos asignaturas al año (Ministerio de Educación, 1995).

Equidad y calidad son aspectos con similar relevancia, y si bien la equidad en Argentina ha aumentado de manera sostenida durante los últimos años (García de Fanelli \& Broto, 2019), se observan, por otro lado, algunas dificultades en cuanto a los indicadores de calidad. Se registra así en los alumnos, una demora en la finalización de sus estudios logrando graduarse en tiempo y forma solo el 30 \% de los inscriptos (Ministerio de Educación, 2019). En esta línea se observa que solo un $15 \%$ de los estudiantes aprueban todas las asignaturas previstas por año, mientras que el 35,7 \% no aprueba ninguna durante el año académico y el 15,3 \% aprueba solo una (Fundación Libertad, 2019). Por último, además del rezago estudiantil, se observa que un número importante de educandos abandona sus estudios alcanzándose una tasa de deserción universitaria del 21 \% (Ministerio de Educación, 2019). Estas dificultades que los estudiantes presentan para permanecer en el sistema universitario y alcanzar el éxito académico, son causadas por una multiplicidad de factores demográficos, ambientales, académicos, pedagógicos y psicoló- 
gicos, entre otros. Por esta razón, es que varios trabajos se han focalizado en el análisis de estas variables con el fin de mejorar la calidad de los aprendizajes, y evitar la demora y deserción académica (Alonazi, 2018; Araque et al., 2009; Çizel et al., 2017; Najimi et al., 2013; Stăiculescu \& Elena-Ramona, 2019; Trejo Sánchez, 2019; van Rooij et al., 2017; Yu et al., 2018).

Entre las distintas variables que permiten explicar el rendimiento académico de los estudiantes universitarios se encuentran los enfoques de aprendizaje. La importancia de este constructo se destaca a través de diferentes estudios que verifican su relación con el desempeño estudiantil (García et al., 2019; Kamath et al., 2018; Soffi Puteh et al., 2018; Vivas et al., 2007; Zarooj-Hosseini et al., 2018). Los enfoques de aprendizaje se definen como la combinación de motivos y estrategias que los estudiantes emplean para aprender los contenidos académicos en los diferentes contextos educativos (Biggs, 1989). Se tienen dos tipos de enfoques -Profundo y Superficial- que describen modos de aprendizaje diferentes. El aprendizaje Profundo ocurre cuando los estudiantes manifiestan un real interés por comprender los contenidos académicos y relacionarlos con conocimientos previos. El conocimiento adquirido bajo esta modalidad de aprendizaje alcanza un mayor nivel de consolidación y duración en el tiempo (Kember \& Leung, 1998). Por su parte, el aprendizaje Superficial tiene lugar cuando los estudiantes se interesan por alcanzar los objetivos -aprobar un examen, graduarse- sin importar la comprensión de los contenidos. Este tipo de aprendizaje se da por memorización y reproducción de la información, en lugar de realizar su procesamiento complejo (Biggs, 1989).

Es importante destacar que la metodología de estudio empleada por los alumnos responde a las estrategias que aplican los docentes, de modo que los enfoques de aprendizaje reflejan la manera en que los alumnos perciben el contexto de enseñanza en el cual participan (Biggs, 1988; Entwistle \& Peterson, 2017). De esta manera, el aprendizaje Profundo se encuentra estrechamente relacionado con un tipo de enseñanza basada en su conocimiento previo, la puesta en práctica a través de pruebas de ensayo y error, y la evaluación holística de los contenidos, entre otras cosas (Biggs \& Tang, 2011). Por otro lugar, el aprendizaje Superficial se vincula a un tipo de enseñanza que no demanda de parte del estudiante el procesamiento de los contenidos académicos, sino que por el contrario, este aprende a partir de materiales ya elaborados por los docentes. Según se ha teorizado y verificado empíricamente el enfoque Profundo se asocia positivamente con el éxito académico 
de los estudiantes, mientras que el Superficial lo hace negativamente (Cetin, 2016; Herrmann et al., 2017; Ribeiro et al., 2019).

Por otro lado, el enfoque Profundo se encuentra estrechamente relacionado con la creatividad de los estudiantes, siendo esta capacidad muy valorada en la actualidad dentro del ámbito educativo por distintos organismos internacionales (Mukhopadhyay, 2012). Así, la Comisión Económica para América Latina y el Caribe (CEPAL, 2011), la Organización de Estados Iberoamericanos (OEI, 2010) y la Organización de las Naciones Unidas para la Educación, la Ciencias y la Cultura (UNESCO, 2017) plantean la importancia de transformar la educación tradicional en una educación con base en la estimulación de la capacidad creativa de los estudiantes y la resolución activa de problemas (CEPAL, 2011; OEI, 2010; UNESCO, 2017). La posición adoptada por estos organismos se debe a la necesidad de cubrir la demanda del mercado laboral de profesionales con elevada capacidad creativa y de innovación. Orientar la educación superior hacia una dirección creativa no es una tarea fácil si se considera que la creatividad es un fenómeno multidimensional (Simonton, 2003). Por esta razón, es que se vuelve necesario evaluar primero el constructo para conocerlo y luego tomar decisiones con base en a esos resultados. La creatividad puede evaluarse principalmente de cuatro maneras: a través del ambiente, el producto, el proceso y la persona (López et al., 2006). Si bien, las cuatro categorías revisten igual relevancia, en general las referidas al proceso y a la persona son las mayormente adoptadas para evaluar la creatividad en el ámbito psicoeducativo. En el caso del Proceso Creativo se evalúan las respuestas creativas que dan los sujetos frente a un problema. Para esto se emplean habitualmente los indicadores propuestos por Guilford (1950): fluidez -cantidad-, flexibilidad -variedad-, originalidad -novedad-y elaboración -detalles-. Con respecto a la Personalidad Creativa, si bien hay varios componentes que pueden evaluarse-actitudes, intereses, tendencias perceptivo/cognitivas y biografías, entre otros- uno de los más estudiados en el ámbito educativo es el relacionado estrictamente con aquellos rasgos de personalidad considerados creativos, tales como apertura a la experiencia, sensibilidad, curiosidad, imaginación, individualismo e iniciativa, entre otros (Benlliure, 2006; Santamaría \& Sánchez, 2012; Wang et al., 2017).

La evaluación del Proceso y la Personalidad Creativa arroja información valiosa que puede ser utilizada para promover la creatividad en el ámbito académico a partir de la intervención sobre distintos factores -estrategias educativas, ambiente de aprendizaje- que a su vez coinciden con el tipo de 
aprendizaje Profundo (Liu et al., 2020; Richardson \& Mishra, 2018; Wu et al., 2014). Se busca así incluir nuevas prácticas creativas en el ámbito educativo superior con el fin de formar futuros profesionales con un perfil creativo (Watson, 2018).

A partir de todo lo mencionado, el presente artículo busca analizar los enfoques de aprendizaje y la creatividad en los estudiantes universitarios, y su relación con el rendimiento académico. Para esto se plantean los siguientes objetivos: 1) Analizar diferencias estadísticamente significativas en las variables creativas -Proceso Creativo y Personalidad Creativa-, y los enfoques de aprendizaje -Profundo y Superficial-, según variables sociodemográficas y académicas -nivel educativo alcanzado por el padre y la madre, género y facultad-, 2) Estudiar la influencia de las variables creativas y los enfoques de aprendizaje sobre el rendimiento académico.

Se espera que la información resultante de este estudio permita comprender el modo en que los estudiantes aprenden en el actual contexto de enseñanza-aprendizaje y el lugar que la creatividad ocupa.

\section{MÉTODO}

\subsection{Diseño}

Se empleó un estudio no experimental, transversal, correlacional, de diferencias entre grupos y explicativo. Los datos se recogieron mediante un muestreo por conveniencias.

\subsection{Participantes}

Se seleccionaron estudiantes de instituciones universitarias públicas de entre 17 y 36 años de edad, que al momento de la administración se encontraran cursando en cualquier instancia del trayecto académico. Participaron 301 estudiantes universitarios ( $46,7 \%$ varones y $53,3 \%$ mujeres) de las facultades de Psicología (51 \%) e Ingeniería (49\%) ( $M_{\text {edad }}=21,02 ; D E=3,69$ ).

\subsection{Instrumentos}

\subsubsection{Encuesta sociodemográfica y de datos académicos}

Se recogió información para describir la muestra -género, edad, universidad, facultad, carrera-, y para estimar el rendimiento académico -cantidad de años que el estudiante lleva cursando la carrera y número total de asigna- 
turas aprobadas-. Para el cálculo del desempeño estudiantil se adoptó la medida sugerida por Miguel-Díaz y Arias-Blanco (1999), que, en línea con la Ley 24.521 de Educación Superior de Argentina (Ministerio de Educación, 1995) propone calcular el cociente entre el número de asignaturas aprobadas y la cantidad de años que el estudiante lleva cursando la carrera.

\subsubsection{Escala de Personalidad Creativa}

Se aplicó la versión localmente adaptada del instrumento (FreibergHoffmann et al., 2019; Gough, 1979)siendo la personalidad una de las que mayor relevancia reviste. Se propone así, adaptar localmente la Escala de Personalidad Creativa (EPC). La misma se compone de ocho adjetivos con una escala dicotómica de respuesta en la que los participantes deben contestar para cada uno de ellos si se sienten representados (+) o no (-). La escala permite obtener una medida global de la Personalidad Creativa a partir de la suma de seis de los adjetivos -Inteligente, Con Intereses, Inventivo, Original, Habil, Sexy-y la resta dos -Común, Sin Intereses-. Esta versión local presenta evidencias de validez de constructo -análisis factorial exploratorio y confirmatorio- y de criterio concurrente, como también una adecuada consistencia interna de la dimensión resultante del proceso de adaptación (alfa ordinal=0,77).

\subsubsection{Test CREA (Corbalán-Berná et al., 2006)}

Se trata de una prueba verbal que permite evaluar el Proceso Creativo. Para ello se solicita al evaluado redactar la mayor cantidad de preguntas posibles a partir de una imagen presentada. La tarea tiene un tiempo límite de cuatro minutos. La técnica valora el Proceso Creativo de manera unidimensional obteniendo un puntaje total de la sumatoria de los puntajes parciales otorgados a cada pregunta de acuerdo con el grado de complejidad con el que fueron formuladas. El instrumento se integra por tres láminas diseñadas para ser aplicadas a distintos grupos etarios: CREA A -10 años en adelante-, CREA B -a partir de 12-, y CREA C -desde los 6 años-. Es importante aclarar que las tres láminas son formas paralelas que no necesariamente deben ser aplicadas en su conjunto, sino que pueden utilizarse de manera independiente. Para el presente estudio se empleó la lámina $A$, cuya adaptación local cuenta con evidencias de validez predictiva, convergente y discriminante, como también confiabilidad entre formas paralelas (Corbalán-Berná et al., 2006). 


\subsubsection{Cuestionario Revisado de Procesos de Estudio, Dos Factores}

(R-SPQ-2F) (Biggs et al., 2001)

Se administró la versión del cuestionario adaptado a estudiantes universitarios de Buenos Aires (Freiberg-Hoffmann \& Fernández-Liporace, 2016). El mismo se compone de 20 reactivos que deben ser contestados en una escala Likert de cinco opciones de respuesta que van de 1 -Nada de Acuerdo- a 5 -Muy de Acuerdo-. El instrumento bidimensional permite evaluar los enfoques Profundo y Superficial y cuenta con evidencias de validez de contenido, aparente y de constructo -estudio factorial exploratorio y confirmatorio- y una apropiada consistencia interna de sus dimensiones.

\subsection{Procedimientos}

Los datos fueron recogidos por un psicólogo entrenado, durante el horario de clases. Se contó con el aval de las instituciones participantes y de los profesores a cargo de los cursos. Se administró a los estudiantes un consentimiento informado donde además de explicar en detalle el propósito de la investigación se les informó sobre el carácter anónimo y voluntario de su participación, especificando que no recibirían devoluciones individuales ni retribución económica o académica a cambio de su colaboración.

\subsection{Análisis de Datos}

Se aplicó una prueba MANOVA para analizar el efecto de las variables sociodemográficas y académicas sobre las variables psicológicas -Proceso Creativo, Personalidad Creativa, y enfoques de aprendizaje Profundo y Superficial-. Por otro lado, se empleó un análisis de regresión múltiple para examinar la influencia de las variables psicológicas sobre el rendimiento académico.

\section{RESULTADOS}

Se aplicó en primer lugar el análisis MANOVA tomando como variables dependientes el Proceso Creativo, la Personalidad Creativa y los enfoques de aprendizaje -Profundo y Superficial-. Por otro lado, las variables de nivel educativo alcanzado por el padre y la madre, género y facultad fueron consideradas como factores.

Se comenzó por analizar la igualdad de varianzas y covarianzas de las variables dependientes verificándose su cumplimiento. Dado este resultado se optó por la lectura del estadístico Lambda de Wilkis para examinar las hipótesis subyacentes al análisis en cuestión (Huberty \& Petoskey, 2000). Se verificaron 
así efectos multivariados significativos para las variables género y facultad (Tabla 1).

Tabla 1

\begin{tabular}{ccccccc}
\hline & LAMBDA & $\mathbf{F}$ & $\mathbf{G L}_{\text {HIPÓTESIS }}$ & $\mathbf{G L}_{\text {ERROR }}$ & SIG. & $\mathrm{H}^{2}$ \\
\hline Intersección & 0,023 & 2065,974 & 4 & 192 & 0,000 & 0,977 \\
Género & 0,889 & 5,993 & 4 & 192 & 0,000 & 0,111 \\
Facultad & 0,923 & 4,029 & 4 & 192 & 0,004 & 0,077
\end{tabular}

Una primera lectura de los valores Lambda de las variables género y facultad muestran un efecto parejo de ambas sobre el modelo testeado. Además, puede decirse que si bien se han registrado diferencias estadísticamente significativas, su magnitud es relativamente pequeña ya que los valores de los estadísticos son cercanos a uno (O'Rourke et al., 2005). Para conocer en detalle las diferencias halladas se examinaron los contrastes univariados observándose con respecto a la variable género diferencias estadísticamente significativas en el enfoque Profundo $\left(M_{\text {varones }}=30,95, D E=5,62 ; M_{\text {mujeres }}=32,37\right.$, $D E=5,13 ; p=0,019)$, en el Superficial $\left(M_{\text {varones }}=25,26, D E=6,13 ; M_{\text {mujeres }}=21,34\right.$, $D E=5,37 ; p=0,001)$ y en la Personalidad Creativa $\left(M_{\text {varones }}=4,93, D E=1,88\right.$; $\left.M_{\text {mujeres }}=4,14, D E=1,65 ; p=0,003\right)$.

En relación a la variable facultad se observó una diferencia significativa en el Proceso Creativo a favor de los estudiantes de Psicología $\left(M_{\text {Psicologia }}=13,41\right.$, $\left.D E=4,24 ; M_{\text {Ingenieria }}=12,71, D E=3,99 ; p=0,010\right)$.

Pasando ahora al examen de la influencia de las variables psicológicas sobre el rendimiento académico, se efectuó un análisis de regresión lineal múltiple. Los parámetros se estimaron a través del método de mínimos cuadrados. Además, se empleó el procedimiento por pasos hacia atrás. El rendimiento académico se calculó aplicando el cociente entre la cantidad de asignaturas aprobadas y los años de antigüedad que lleva cada estudiante en la carrera (Miguel-Díaz \& Arias-Blanco, 1999).

Se comenzó por explorar el rendimiento académico (variable dependiente), en busca de valores extremos que pudieran afectar los resultados. Esta instancia no registró casos atípicos. Posteriormente, de los modelos arrojados por este procedimiento se seleccionó, por su ajuste y parsimonia, uno que conservó solo dos variables independientes (Proceso Creativo y enfoque Superficial). Al analizar la bondad de ajuste del modelo se observó un tamaño del efecto entre bajo y moderado $(R 2=0,101)$. A su vez el porcentaje de varianza total explicada por el modelo (R2corregida) fue del 9,1\%, y la magnitud del error 
típico fue de 1,55, ubicándose por debajo del límite aceptable para un nivel de significación del 5 \% (Álvarez-Cáceres, 1995; Gutiérrez, 2007). Asimismo, el análisis de varianza (ANOVA) verifica la relación lineal entre la variable dependiente y las independientes $(p<0,01)$.

Yendo ahora al análisis del efecto de las variables independientes sobre la dependiente, se tiene en primer lugar al estadístico B que informa que por cada punto en que el enfoque Superficial decrece y el Proceso Creativo aumenta, el rendimiento académico mejora en 0,073 y 0,076 puntos respectivamente. Además, se observa que el $27,6 \%$ de la variabilidad del enfoque Superficial y el $18,5 \%$ del Proceso Creativo se encuentran relacionados con la explicación del rendimiento académico (Tabla 2).

Tabla 2

\begin{tabular}{ccccccc}
\hline MODELO & B & ERROR TíPICO & BETA & T & SIG. & IC 95 \% \\
\hline (Constante) & 3,203 & 0,557 & & 5,748 & 0,000 & {$[2,103,4,302]$} \\
Enfoque Superficial & $-0,073$ & 0,019 & $-0,276$ & $-3,904$ & 0,000 & {$[-0,109,-0,036]$} \\
Proceso Creativo & 0,076 & 0,029 & 0,185 & 2,612 & 0,010 & {$[0,019,0,134]$} \\
\hline
\end{tabular}

\section{DISCUSIÓN}

El presente trabajo analiza los enfoques de aprendizaje y la creatividad en estudiantes universitarios con el fin de identificar aquellas variables que, además de contribuir con la mejora del rendimiento académico, son actualmente valoradas de manera positiva tanto en el ámbito educativo, como laboral. En primera instancia, se analizan los enfoques de aprendizaje Profundo y Superficial- y la creatividad -Proceso Creativo y Personalidad Creativa- en relación con variables sociodemográficas y académicas de los estudiantes registrándose algunas diferencias. Así se observa que las mujeres, a diferencia de los varones, se encuentran motivadas intrínsecamente a la hora de aprender los contenidos académicos. De este modo puede decirse que ellas poseen un elevado nivel conceptual que les permite abordar las tareas de manera significativa, intentando comprender los conceptos novedosos a la luz del conocimiento previo. Tienen una marcada preferencia por aprender los contenidos de manera holística, en lugar de enfocarse en detalles de manera aislada. Por su parte, los varones, a diferencia de las mujeres, manifiestan una marcada motivación extrínseca hacia el aprendizaje guiado por el interés de aprobar las asignaturas sin importar la comprensión de contenidos. Estos alumnos presentan una ansiedad elevada, y no suelen 
tener demasiado tiempo para destinar al estudio ya que suelen realizar varias actividades extra-académicas (Biggs \& Tang, 2011).

Por otro lado, se observa que los varones a diferencia de las mujeres poseen una mayor Personalidad Creativa, autopercibiéndose como sujetos con múltiples intereses, inteligentes, inventivos, originales y hábiles. Este resultado parecería ser contradictorio con el obtenido en los enfoques de aprendizaje, ya que la creatividad suele asociarse con el enfoque Profundo, y no con el Superficial (Miller \& Dumford, 2016; Rogaten et al., 2012). Una posible explicación podría realizarse a partir de la Escala de Personalidad Creativa, y el rasgo de personalidad que esta evalúa vinculado con la amplitud de intereses. Este rasgo ha sido el que mayor poder explicativo ha demostrado poseer, en comparación con el resto, en el estudio de validación local del instrumento (Freiberg-Hoffmann et al., 2019), siendo la personalidad una de las que mayor relevancia reviste. Se propone así, adaptar localmente la Escala de Personalidad Creativa (EPC). Se considera a la multiplicidad de intereses como una característica de gran relevancia para el desarrollo de la creatividad en los individuos, ya que ello les posibilita adquirir información a partir de una diversidad de fuentes, para luego combinarla de manera atípica dando lugar a productos creativos (Csikszentmihalyi, 1996). Esta característica se condice con la descripción del enfoque Superficial acerca de la realización de actividades extra-académicas por sobre las académicas. De este modo, podría hipotetizarse que el estudiante varón tendría varios intereses que lo llevarían a adoptar un tipo de enfoque Superficial durante las tareas académicas. No obstante, el enfoque Profundo también tiene un rol fundamental, ya que posibilitaría al estudiante alcanzar los niveles de articulación conceptual necesarios para dar lugar a los productos creativos. Así, podría hipotetizarse que ambos componentes son relevantes en relación a la creatividad, y no solo el tipo de aprendizaje Profundo. Con base en este resultado se continuará trabajando a fin de conocer la medida en que ambos enfoques se relacionan con la Personalidad Creativa de los estudiantes y cómo ello puede impactar en sus actividades académicas.

Con respecto al análisis de las variables según facultad se observa que los estudiantes de Psicología, a diferencia de los de Ingeniería, tienen una capacidad más elevada para generar un mayor número de ideas -fluidez-, diferentes entre sí -flexibilidad-, novedosas -originalidad-, coherentes y atractivas -elaboración- (Corbalán-Berná et al., 2006). Esta diferencia a favor de los estudiantes de Psicología podría explicarse a partir de la naturaleza verbal del instrumento que se empleó para medir la creatividad. 
Otros estudios, que también analizan la creatividad a nivel verbal, tampoco registran resultados favorables a los estudiantes de Ingeniería (Chiecher et al., 2018; Islas-Torres et al., 2019). No obstante, hallazgos reportados por otros trabajos, sí dan cuenta de la presencia de un tipo de creatividad en los estudiantes de Ingeniería vinculada a formas de razonamiento no verbal y distintas clases de acciones creativas (Ayob et al., 2012; Elisondo et al., 2018). De este modo, sería interesante que en el futuro se apliquen otros instrumentos que permitan evaluar el Proceso Creativo a partir de distintos tipos de estímulos -lógicos, gráficos, mecánicos, entre otros-, posibilitando describir mejor el fenómeno en diferentes especialidades académicas.

Pasando ahora al examen de la influencia que tienen los enfoques de aprendizaje y la creatividad sobre el rendimiento académico de los estudiantes, se observa que el modelo extraído conserva como variables explicativas el enfoque Superficial y el Proceso Creativo. El primero se relaciona negativamente con el rendimiento académico, mientras que el segundo lo hace positivamente, coincidiendo este resultado con el reportado por otros estudios (De la Fuente Arias et al., 2013; Gajda et al., 2017; Herrmann et al., 2017). Estos hallazgos muestran que el enfoque Superficial se encuentra más implicado con el desempeño estudiantil que el Proceso Creativo, ya que aporta mayor porcentaje de su variabilidad a la explicación del rendimiento. Este dato resulta interesante, ya que podría hipotetizarse que la creatividad en el ámbito universitario, si bien colabora con el éxito de los estudiantes, tiene una baja participación en el proceso de aprendizaje. Se plantea, así, la necesidad de diseñar prácticas educativas y pedagógicas orientadas a la estimulación de la creatividad en los educandos a partir de actividades que promuevan los procesos productivos. Para ello, se requiere un ambiente desestructurado y la estimulación de un tipo de motivación creativa que fomente la curiosidad, la generación de esquemas cognitivos, el deseo de crear, la autoafirmación y la reestructuración cognitiva, entre otras cosas (Corbalán-Berná et al., 2006). Por otra parte, debe mencionarse que el enfoque Profundo no realizó un aporte significativo a la explicación del rendimiento académico. Esto estaría indicando que el contexto educativo -estrategias de enseñanza y modalidades de evaluación- en el que participan los estudiantes no estaría demandando por parte de estos un aprendizaje que requiera el empleo de un procesamiento cognitivo complejo de los contenidos académicos. Tomando en cuenta este resultado debería plantearse la necesidad de modificar las estrategias educativas en conjunto con las modalidades de evaluación, a fin de promover en los estudiantes estrategias y motivos profundos que den lugar a aprendizajes más complejos y duraderos. 
La investigación presenta algunas limitaciones que merecen destacarse. Por un lado, la muestra analizada solo incluye estudiantes de dos carreras universitarias, restringiendo así la generalización de los resultados. Se planea continuar analizando las variables incluidas en este diseño en una muestra más heterogénea, a fin de ampliar la descripción de las características de los estudiantes a distintas disciplinas académicas. Otra limitación que tiene el estudio es la referida a la medida empleada para evaluar el rendimiento académico. Como se sabe, el desempeño de los estudiantes constituye una variable compleja, siendo toda aproximación a la misma una estimación relativa y parcial (Martín et al., 2008). De este modo, se espera poder incluir indicadores adicionales que permitan estimar una medida más precisa del fenómeno.

\section{CONCLUSIONES}

Como se ha mencionado, la metodología de estudio que los alumnos utilizan para aprender contenidos novedosos se encuentra estrechamente relacionada con el tipo de contexto en el que participan los educandos y el modo en que estos lo perciben (Biggs, 1988; Entwistle \& Peterson, 2017). Desde este punto de vista, se vuelve necesario repensar las estrategias de enseñanza y la metodología de evaluación de contenidos para lograr equilibrar los enfoques de aprendizaje empleados por los estudiantes, fundamentalmente si el enfoque Superficial puede llegar a tener efectos negativos sobre el rendimiento estudiantil. En este sentido, no se plantea la necesidad de disminuir, o incluso anular, la presencia de un enfoque -Superficial- en detrimento del incremento de otro -Profundo-, sino que por el contrario se debe buscar alcanzar una convivencia entre ambos que posibilite a los estudiantes mejorar la calidad de sus aprendizajes (Entwistle, 2000). Asimismo, si se consideran los resultados de estudios anteriores (Heinstrom, 2000; Mukhopadhyay, 2012), y los hallazgos obtenidos en el presente trabajo es posible concluir que el equilibrio entre los enfoques de aprendizaje colaboraría con el desarrollo de la creatividad en los estudiantes. Por esta razón, es que deben adoptarse decisiones pedagógicas para poder mejorar los aprendizajes estudiantiles y al mismo tiempo formar futuros profesionales que den soluciones creativas a los problemas.

En cuanto a las limitaciones que presenta este trabajo, sería de gran utilidad recoger más información sociodemográfica y académica con el fin de controlar su potencial efecto sobre los resultados. También debe destacarse el posible sesgo de respuesta de los evaluados relacionado con la fatiga, ya que la administración de las pruebas se efectuó al finalizar cada clase. En este 
sentido, para evitar este efecto, será importante recolectar los datos como primera actividad áulica. Estos aspectos serán tenidos en cuenta en futuros estudios.

Conflicto de interés: Los autores no presentan conflicto de interés.

Financiamiento: El trabajo ha sido financiado por la Universidad de Buenos Aires (UBACYT 20020170100064BA) y el Consejo Nacional de Investigaciones Cientificas y Técnicas (CONICET).

Agradecimientos: Los autores agradecen a las instituciones que participaron en el estudio.

\section{REFERENCIAS}

Alonazi, A. A. (2018). Psychological, academic and demographic variables affecting students' academic achievement among first year college students in Saudi Arabia (University of the Pacific). University of the Pacific. https://search.proquest.com/ openview/fd3b1b47e384669500ee7c0ea8ad0471/1?pq-origsite=gscholar\&c$\mathrm{bl}=18750 \&$ diss $=\mathrm{y}$

Álvarez Cáceres, R. (1995). Estadística multivariante y no paramétrica con SPSS. Díaz de Santos.

Araque, F., Roldán, C., \& Salguero, A. (2009). Factors influencing university drop out rates. Computers and Education, 53(3), 563-574. https://doi.org/10.1016/j. compedu.2009.03.013

Ayob, A., Hussain, A., Mustaffa, M. M., \& Majid, R. A. (2012). Assessment of Creativity in Electrical Engineering. Procedia - Social and Behavioral Sciences, 60, 463-467. https://doi.org/10.1016/j.sbspro.2012.09.407

Benlliure, V. (2006). Evaluar aspectos de la personalidad. En S. De la Torre \& V. Violant (Eds.), Comprender y evaluar la creatividad (pp. 303-323). Aljibe.

Biggs, J. B. (1988). Assessing student approaches to learning. Australian Psychologist, 23(2), 197-206. https://doi.org/10.1080/00050068808255604

Biggs, J. B. (1989). Approaches to the Enhancement of Tertiary Teaching. Higher Education Research \& Development, 8(1), 7-25. https://doi. org/10.1080/0729436890080102

Biggs, J., Kember, D., \& Leung, D. Y. P. (2001). The revised two-factor Study Process Questionnaire: R-SPQ-2F. The British Journal of Educational Psychology, 71, 133-149. https://doi.org/10.1348/000709901158433

Biggs, J. \& Tang, C. (2011). Teaching for quality learning at university. McGraw-Hill.

CEPAL (2011). Educación, desarrollo y ciudadanía en América Latina. CEPAL.

Cetin, B. (2016). Approaches to learning and age in predicting college students' academic achievement. Journal of College Teaching \& Learning (TLC), 13(1), 21-28. https://doi.org/10.19030/tlc.v13i1.9568

Chiecher, A. C., Elisondo, R. C., Paoloni, P.V., \& Donolo, D. S. (2018). Creatividad, géneroy rendimiento académico en ingresantes de ingeniería. Revista Iberoamericana de Educación Superior, 9(24), 138-151. https://doi.org/10.22201/iisue.20072872e.2018.24.269

Çizel, B., Ajanovic, E., \& Çizel, R. (2017). Effects of Psychological and Demographic Factors on Academic Performance. Mediterranean Journal of Humanities, 7(2), 133-147. https://doi.org/10.13114/MJH.2017.353 
Corbalán-Berná, J., Martínez-Zaragoza, F., Donolo, D. S., Alonso-Monreal, C., Tejerina-Arreal, M., \& Limiñana-Gras, R. M. (2006). CREA. Inteligencia Creativa. TEA.

Csikszentmihalyi, M. (1996). Creatividad. Paidós.

De la Fuente Arias, J., Sander, P., \& Putwain, D. (2013). Relationship between Undergraduate Student Confidence, Approach to Learning and Academic Performance: The role of gender // Relación entre la confianza académica, los enfoques de aprendizaje y el rendimiento académico de estudiantes universitarios... Revista de Psicodidactica / Journal of Psychodidactics, 18(2), 375-393. https://doi. org/10.1387/RevPsicodidact.7078

Elisondo, R. C. Chiecher, A. C. \& Paoloni, P. V. R. (2018). Creatividad, ocio y rendimiento académico en estudiantes de Ingeniería. ReiDoCrea, 7, 28-42. https:// www.ugr.es/ reidocrea/7-3.pdf

Entwistle, N. (2000). Promoting deep learning through teaching and assessment. Paper presented at AAHE Assessment Conference, North Carolina, Estados Unidos. https://www.researchgate.net/publication/285909232_Promoting deep_learning_through_teaching_and_assessment

Entwistle, N., \& Peterson, E. (2017). Learning Styles and Approaches to Studying. In Reference Module in Neuroscience and Biobehavioral Psychology. Elsevier. https://doi.org/10.1016/B978-0-12-809324-5.05623-6

Freiberg-Hoffmann, A., \& Fernández-Liporace, M. M. (2016). Enfoques de Aprendizaje en Estudiantes Universitarios Argentinos según el R-SPQ-2F: Análisis de sus Propiedades Psicométricas. Revista Colombiana de Psicologia, 25(2), 307-329. https://doi.org/10.15446/rcp.v25n2.51874

Freiberg-Hoffmann, A., Vigh, C., \& Fernández-Liporace, M. (2019). Adaptación de la Escala de Personalidad Creativa en estudiantes universitarios de Argentina. Anales de Psicología, 35(2), 290-299. https://doi.org/10.6018/analesps.35.2.346131

Gajda, A., Karwowski, M., \& Beghetto, R. A. (2017). Creativity and academic achievement: A meta-analysis. Journal of Educational Psychology, 109(2), 269-299. https://doi.org/10.1037/edu0000133

García de Fanelli, A., \& Broto, A. (2019). Financiamiento de las universidades nacionales en la Argentina: principales indicadores y tendencias. Faces, 25(53), 51-70. http://nulan.mdp.edu.ar/3254/1/FACES-53-gdefanelli-broto.pdf

García, M. I. B., Lamos Duarte, A. F., Vargas Rivera, O. I., Camargo Villalba, G. E., \& Capacho, N. S. (2019). Enfoques de aprendizaje, rendimiento académico y factores relacionados en estudiantes que cursan último año de los programas de la Facultad de Ciencias de la Salud. Educación Médica, 20, 10-17. https://doi. org/10.1016/j.edumed.2017.11.008

Gough, H. G. (1979). A creative personality scale for the Adjective Check List. Journal of Personality and Social Psychology, 37(8), 1398-1405. https://doi. org/10.1037/0022-3514.37.8.1398

Guilford, J. P. (1950). Creativity. American Psychologist, 5(9), 444-454. https://doi. org/10.1037/h0063487

Gutiérrez, J. C. A. (2007). Estadística general aplicada. Fondo Editorial Universidad EAFIT.

Harvey, L., \& Green, D. (1993). Defining Quality. Assessment \& Evaluation in Higher Education, 18(1), 9-34. https://doi.org/10.1080/0260293930180102

Heinstrom, J. (2000). Impact of personality and approaches to learning on information behavior. Information Research, 5(3), 213-219. http://informationr.net/ ir/5-3/paper78.html 
Herrmann, K. J., McCune, V., \& Bager-Elsborg, A. (2017). Approaches to learning as predictors of academic achievement: Results from a large scale, multi-level analysis. Högre Utbildning, 7(1), 29-42. https://doi.org/10.23865/hu.v7.905

Huberty, C. J., \& Petoskey, M. D. (2000). Multivariate Analysis of Variance and Covariance. In Handbook of Applied Multivariate Statistics and Mathematical Modeling (pp. 183-208). Elsevier. https://doi.org/10.1016/B978-012691360$6 / 50008-2$

Islas Torres, C., Carranza Alcántar, M. D. R., Pérez Poch, A., \& Salán Ballesteros, N. (2019). Estudio sobre la creatividad relacionada con la habilidad de programadores universitarios. Revista Electrónica de Investigación Educativa, 21(1), 1. https://doi.org/10.24320/redie.2019.21.e34.2143

Kamath, A., Rao, R., Shenoy, P. J., \& Ullal, S. D. (2018). Approaches to learning and academic performance in pharmacology among second-year undergraduate medical students. Scientia Medica, 28(4), 32395. https://doi.org/10.15448/19806108.2018.4.32395

Kember, D., \& Leung, D. Y. P. (1998). The dimensionality of approaches to learning: an investigation with confirmatory factor analysis on the structure of the SPQ and LPQ. British Journal of Educational Psychology, 68(3), 395-407. https://doi. org/10.1111/j.2044-8279.1998.tb01300.x

Libertad, F. (2019). Radiografías de las universidades argentinas. https://libertad.org. ar/web/wp-content/uploads/2019/09/CISE_Radiografia_Universidades.pdf

Liu, H. Y., Wang, I. T., Chen, N. H., \& Chao, C. Y. (2020). Effect of creativity training on teaching for creativity for nursing faculty in Taiwan: A quasi-experimental study. Nurse Education Today, 85, 104231. https://doi.org/10.1016/j.nedt.2019.104231

López, O., Corbalán, F. J., \& Martínez, F. (2006). Instrumentos y medidas clásicas de la creatividad. En S. De la Torre \& V. Violant (Eds.), Comprender y evaluar la creatividad (pp. 213-236). Aljibe.

Martín, E., García, L. A., Torbay, Á., \& Rodríguez, T. (2008). Estrategias de aprendizaje y rendimiento académico en estudiantes universitarios. International Journal of Psychology and Psychological Therapy, 8(3), 401-412. https://doi. org/10.21676/2389783X.661

Miguel Díaz, F., \& Arias Blanco, J. (1999). La evaluación del rendimiento inmediato en la enseñanza universitaria. Revista de Educación, (320), 353-377. http:// www.educacionyfp.gob.es/dam/jcr:6093eeea-569d-4615-86be-60fcc277d804/ re3201707982-pdf.pdf

Miller, A. L., \& Dumford, A. D. (2016). Creative Cognitive Processes in Higher Education. The Journal of Creative Behavior, 50(4), 282-293. https://doi.org/10.1002/jocb.77

Ministerio de Educación, S. de P. U. (2019). Síntesis de información estadísticas universitarios 2018-2019. https://www.argentina.gob.ar/sites/default/files/ sintesis_2018-2019_sistema_universitario_argentino_0.pdf

Ministerio de Educación. (1995). Ley No 24.521 de Educación Superior. Ministerio de Educación de la Nación Argentina.

Mukhopadhyay, D. R. (2012). Investigation of Creativity in Physics in the Context of Learning in Association with Deep Approach to Study. IOSR Journal of Humanities and Social Science, 4(2), 24-30. https://doi.org/10.9790/0837-0422430

Najimi, A., Sharifirad, G., Amini, M., \& Meftagh, S. (2013). Academic failure and students' viewpoint: The influence of individual, internal and external organizational factors. Journal of Education and Health Promotion, 2(1), 22. https://doi. org/10.4103/2277-9531.112698

Organización de Estados Iberoamericanos (OEI, 2010). Ciencia, tecnología y universidad en Iberoamérica. Eudeba. 
O'Rourke, N., Hatcher, L., \& Stepanski, E. (2005). Using SAS for univariate \& multivariate statistics. SAS Institute Inc.

Ribeiro, L., Rosário, P., Núñez, J. C., Gaeta, M., \& Fuentes, S. (2019). First-Year Students Background and Academic Achievement: The Mediating Role of Student Engagement. Frontiers in Psychology, 10. https://doi.org/10.3389/ fpsyg.2019.02669

Richardson, C., \& Mishra, P. (2018). Learning environments that support student creativity: Developing the SCALE. Thinking Skills and Creativity, 27, 45-54. https:// doi.org/10.1016/j.tsc.2017.11.004

Rogaten, J., Moneta, G., \& Spada, M. (2012). A trait-state model of deep approach to studying in university students. Paper Presented at 3rd International Conference on Education and Educational Psychology. Istanbul, Turkey. http://oro.open. ac.uk/47888/

Santamaría, H., \& Sánchez, R. (2012). Creatividad y rasgos de personalidad en estudiantes universitarios: estudio transversal de asociación. Revista Colombiana de Psiquiatría, 41(2), 284-298. https://doi.org/10.1016/S0034-7450(14)60005-6

Simonton, D. K. (2003). Scientific creativity as constrained stochastic behavior: The integration of product, person, and process perspectives. Psychological Bulletin, 129(4), 475-494. https://doi.org/10.1037/0033-2909.129.4.475

Soffi Puteh, M., Abdul Latif, N. E., Mansor, N., Hamid, Y., Halid, S., \& Ghazali, Z. (2018). Learning Approaches and Academic Performance. The Journal of Social Sciences Research, (SPI6), 746-752. https://doi.org/10.32861/jssr.spi6.746.752

Stăiculescu, C., \& Elena Ramona, R. N. (2019). University dropout. Causes and solution. Mental Health: Global Challenges Journal, 1(1), 71-75. https://doi. org/10.32437/mhgcj.v1i1.29

Trejo Sánchez, K. (2019). Outline of an Integrative Evaluation of the Main Factors That. Editorial. Revista Boletín Redipe, 8(10), 24-34. https://dialnet.unirioja.es/ descarga/articulo/7528321.pdf

UNESCO. (2013). Situación educativa de América Latina y el Caribe: Hacia la educación de calidad para todos al 2015. Orealc/Unesco. http://www.unesco.org/new/ fileadmin/MULTIMEDIA/FIELD/Santiago/images/SITIED-espanol.pdf

UNESCO. (2017). Informe de seguimiento de la educación en el mundo 2016. París.

Van Rooij, E. C. M., Jansen, E. P. W. A., \& Van de Grift, W. J. C. M. (2017). Factors that contribute to secondary school students' self-efficacy in being a successful university student. Research in Post-Compulsory Education, 22(4), 535-555. https://doi.org/10.1080/13596748.2017.1381301

Vivas, R., Lastra, X., \& Yépes, B. (2007). Enfoques de aprendizaje y rendimiento académico universitario en Ecuador. Revista Enlace Universitario, 16, 23-30. https://doi.org/oid=179922664005

Wang, M.-Z., Chen, W., Zhang, C., \& Deng, X.-L. (2017). Personality types and scholarly creativity in undergraduate students: The mediating roles of creative styles. Personality and Individual Differences, 105, 170-174. https://doi.org/10.1016/j. paid.2016.09.050

Watson, J. (2018). Deferred creativity: Exploring the impact of an undergraduate learning experience on professional practice. Teaching and Teacher Education, 71, 206-213. https://doi.org/10.1016/j.tate.2017.12.018

Wu, H.-Y., Wu, H.-S., Chen, I.-S., \& Chen, H.-C. (2014). Exploring the critical influential factors of creativity for college students: A multiple criteria decision-making approach. Thinking Skills and Creativity, 11, 1-21. https://doi.org/10.1016/j. tsc.2013.09.004 
Yu, L., Daniel, D. T., \& Zhu, X. (2018). The influence of personal well-being on learning achievement in university students over time: Mediating or moderating effects of internal and external university engagement. Frontiers in Psychology, 8(JAN), 1-16. https://doi.org/10.3389/fpsyg.2017.02287

Zarooj-Hosseini, R., Enayatrad, M., Ebrahimi, H., Raei, M., Zamani, S., \& Sanabria, M. S. (2018). Investigating the relationship between learning approaches and academic achievement among the students of Shahroud university of medical sciences. International Journal of Health Studies, 4(4), 33-37. http://webcache. googleusercontent.com/search?q=cache:DTBOuVYqKOoJ:ijhs.shmu.ac.ir/index. php/ijhs/article/view/664/448+\&cd=1\&hl=es-419\&ct=clnk\&gl=ar

Esta obra está bajo: Creative commons attribution 4.0 international license. El beneficiario de la licencia tiene el derecho de copiar, distribuir, exhibir y representar la obra y hacer obras derivadas siempre y cuando reconozca y cite la obra de la forma especificada por el autor o el licenciante.

\section{(cc) $\mathbf{B Y}$}

